\title{
Preparing for translocations of a Critically Endangered petrel through targeted monitoring of nest survival and breeding biology
}

\author{
Johannes H. Fischer, Heiko U. Wittmer, Graeme A. Taylor \\ IGOR DEBSKI and DOUg P. ARMSTRONG
}

\begin{abstract}
The population of the recently-described Whenua Hou diving petrel Pelecanoides whenuahouensis comprises c. 200 adults that all breed in a single $0.018 \mathrm{~km}^{2}$ colony in a dune system vulnerable to erosion. The species would therefore benefit from the establishment of a second breeding population through a translocation. However, given the small size of the source population, it is essential that translocations are informed by carefully targeted monitoring data. We therefore modelled nest survival at the remaining population in relation to potential drivers (distance to sea and burrow density of conspecifics and a competitor) across three breeding seasons with varying climatic conditions as a result of the southern oscillation cycle. We also documented breeding phenology and burrow attendance, and measured chicks, to generate growth curves. We estimated egg survival at 0.686 , chick survival at 0.890 , overall nest survival at 0.612 , and found no indication that nest survival was affected by distance to sea or burrow density. Whenua Hou diving petrels laid eggs in mid October, eggs hatched in late November, and chicks fledged in mid January at c. $86 \%$ of adult weight. Burrow attendance (i.e. feeds) decreased from 0.94 to 0.65 visits per night as chicks approached fledging. Nest survival and breeding biology were largely consistent among years despite variation in climate. Nest survival estimates will facilitate predictions about future population trends and suitability of prospective translocation sites. Knowledge of breeding phenology will inform the timing of collection of live chicks for translocation, and patterns of burrow attendance combined with growth curves will structure hand-rearing protocols. A tuhinga whakarāpopoto (te reo Māori abstract) can be found in the Supplementary material.
\end{abstract}

JOHANNES H. FisCHeR (Corresponding author, (1) orcid.org/0000-0003-35271671) School of Biological Sciences, Victoria University of Wellington, PO Box 600, Wellington, 6140, New Zealand. E-mail johannesfischer@live.nl

Неiко U. WiтTMER School of Biological Sciences, Victoria University of Wellington, Wellington, New Zealand

Graeme A. Taylor and Igor DebsKi Aquatic Unit, Department of Conservation, Wellington, New Zealand

Doug P. Armstrong Wildlife Ecology Group, Massey University, Palmerston North, New Zealand

Received 28 April 2020. Revision requested 17 June 2020.

Accepted 31 July 2020. First published online 29 April 2021.
Keywords Aotearoa New Zealand, Bayesian inference, conservation, Pelecanoides whenuahouensis, phenology, Procellariiformes, restoration, Whenua Hou diving petrel

Supplementary material for this article is available at doi.org/10.1017/So030605320000794

\section{Introduction}

Ceabirds, and petrels in particular, are among the most $\checkmark$ threatened taxa (Croxall et al., 2012; Dias et al., 2019). Nearly half of all 125 petrel species (i.e. families Procellariidae, Oceanitidae, Hydrobatidae and Pelecanoididae) are threatened with extinction. Petrel species are affected by a wide range of threats (Dias et al., 2019). On land, petrels are threatened by invasive predators (Jones et al., 2008; Dias et al., 2019), extreme weather events (Cole, 2004; Rodríguez et al., 2019) and light pollution (Rodríguez et al., 2017). At-sea threats include changes in oceanic productivity, climate patterns, and fisheries impacts such as bycatch and competition (Anderson et al., 2011; Zydelis et al., 2013; Grémillet et al., 2018). Various life-history traits render petrels disproportionally vulnerable: they are extremely wideranging (i.e. they utilize entire ocean basins; Shaffer et al., 2006), K-strategists (i.e. low fecundity, delayed sexual maturity, high longevity; Rodríguez et al., 2019) and placed at high trophic levels (i.e. they are top predators; Einoder, 2009). As petrels provide important ecosystem services (e.g. nutrient cycling, bioturbation and seed dispersal; Ellis, 2005; Orwin et al., 2016; Otero et al., 2018), their conservation is a priority.

Translocations are an increasingly common conservation management strategy (Seddon et al., 2007, 2014), including for petrels (Miskelly et al., 2009). A conservation translocation entails the intentional movement of individuals for species recovery or ecosystem restoration (Seddon et al., 2014). Translocations may be effective conservation interventions if habitat is available outside a species' current range, if the species is unlikely to naturally colonize that habitat, and if the translocation is unlikely to cause undesirable impacts. Translocations may involve supporting existing populations (i.e. reinforcement), reinstating populations within the species' indigenous range (i.e. reintroduction), or creating new populations outside of the species' indigenous 
range (i.e. assisted colonization; IUCN, 2013). As petrels are often threatened and facilitate ecosystem functioning, their translocation can be motivated by both species recovery and restoration goals (Miskelly et al., 2009; Jones \& Kress, 2012). For example, Gould's petrels Pterodroma leucoptera have been translocated to Boondelbah Island, Australia, within their indigenous range to strengthen the small existing population (reinforcement; Priddel et al., 2006). Common diving petrels Pelecanoides urinatrix have been translocated to Mana Island, Aotearoa New Zealand, to reinstate the ecosystem functions they once provided (reintroduction; Miskelly et al., 2009).

Poor understanding of the agents of decline can cause translocation failure, including for petrels (Jones \& Kress, 2012; Osborne \& Seddon, 2012). Insights into the drivers of nest survival are key for translocations. Many seabirds, including most petrels, are wide-ranging (e.g. Shaffer et al., 2006). Because of these wide foraging ranges, associated threats at sea are unlikely to be affected by translocation, as has been shown for short-tailed albatrosses Phoebastria albatrus (Deguchi et al., 2014; Orben et al., 2018). Understanding drivers of nest survival at source sites may thus be key to predicting nest survival at potential translocation sites and, consequently, translocation success (Osborne \& Seddon, 2012). As nest survival in seabirds can be subject to interannual fluctuations driven by climatic conditions (Chastel et al., 1995; Quillfeldt et al., 2007), multi-year studies of nest survival are critical.

Poor understanding of the breeding biology of the target species is also a potential cause of translocation failure (Jones \& Kress, 2012). Petrels exhibit high philopatry and their semi-precocial chicks are believed to imprint on their natal colonies prior to fledging (Priddel et al., 2006; Miskelly et al., 2009). Thus, the use of chicks, 1-6 weeks prior to fledging, is required to successfully translocate these species (Miskelly et al., 2009; Jones \& Kress, 2012). As these chicks then need to be hand-reared at the translocation site, detailed information on the breeding biology of the target species is essential to design protocols. For example, data on breeding phenology (i.e. timing and duration of courtship, incubation, guard, and post-guard stages) will inform translocation timing. Data on feeding regimes and chick growth curves will inform hand-rearing regimes.

The Critically Endangered Whenua Hou diving petrel Pelecanoides whenuahouensis is a recently-described burrowing petrel species that could benefit from translocations (Fischer et al., 2018b,c). These birds were once widespread throughout southern Aotearoa New Zealand, but following local extinctions caused by invasive predators (e.g. rats Rattus spp.), the species only survives at a single location: Whenua Hou (Codfish Island; Worthy, 1998; Holdaway et al., 2003; Wood \& Briden, 2008). Here, only 194-208 adults remain in a single colony (Fischer et al., 2020a). Invasive predators have been eradicated from Whenua Hou (McClelland,
2002), but additional threats may still be inhibiting population recovery (Fischer et al., 2020a). Unlike other petrels, the species breeds exclusively in fragile foredunes $<20 \mathrm{~m}$ from the springtide line (Fischer et al., 2018c). Storms and storm surges, as well as climate change, may thus be the main threats to this species (Cole, 2004; Vousdoukas et al., 2020). Competition with the more aggressive common diving petrel for burrow sites may also inhibit population recovery (Fischer et al., 2017). An unsuccessful hybridization attempt between a Whenua Hou diving petrel and a common diving petrel has been recorded (Fischer et al., 2018c), suggesting additional pressures from this closely related species. As common diving petrels appear to be also attracted to Whenua Hou diving petrel calls, acoustic attraction systems may not be an option to establish new Whenua Hou diving petrel colonies (Fischer et al., 2020b). Therefore, a translocation of Whenua Hou diving petrels to a more suitable site could help ensure long-term viability. Detailed information on the factors affecting nest survival and breeding biology is required to meaningfully assess site suitability and design translocation protocols. Such information was previously unavailable.

To inform future translocations, we monitored Whenua Hou diving petrel burrows across three breeding seasons (2017-2019) with a burrowscope, stick palisades, and nest boxes. We aimed to quantify nest survival and its underlying drivers. In addition, we documented breeding phenology and patterns of burrow attendance (as a proxy for feeding regimes), and measured chicks, to generate growth curves.

\section{Study area}

The entire Whenua Hou diving petrel colony is restricted to a $0.018 \mathrm{~km}^{2}$ strip of coastal sand dunes on Whenua Hou (Codfish Island; Fig. 1), $3 \mathrm{~km}$ off the west coast of Rakiura (Stewart Island), Aotearoa New Zealand. This area holds c. 100 Whenua Hou diving petrel burrows (Fischer et al., 2020a). A small number of common diving petrels breed within the study area (Fischer et al., 2017, 2018c).

\section{Methods}

\section{Nest survival}

To quantify Whenua Hou diving petrel nest survival (i.e. egg, chick, and overall nest survival), we monitored $62-78$ burrows $(65-79 \%$ of the total population) from early September to late January during the 2017, 2018 and 2019 breeding seasons (we report the year in which breeding commenced). Monitoring was conducted with a burrowscope (Sextant Technologies, Wellington, Aotearoa New Zealand; Plate 1). Monitoring included daily checks of the burrows monitored for breeding phenology (see below) and weekly checks of all other burrows that allowed access. 


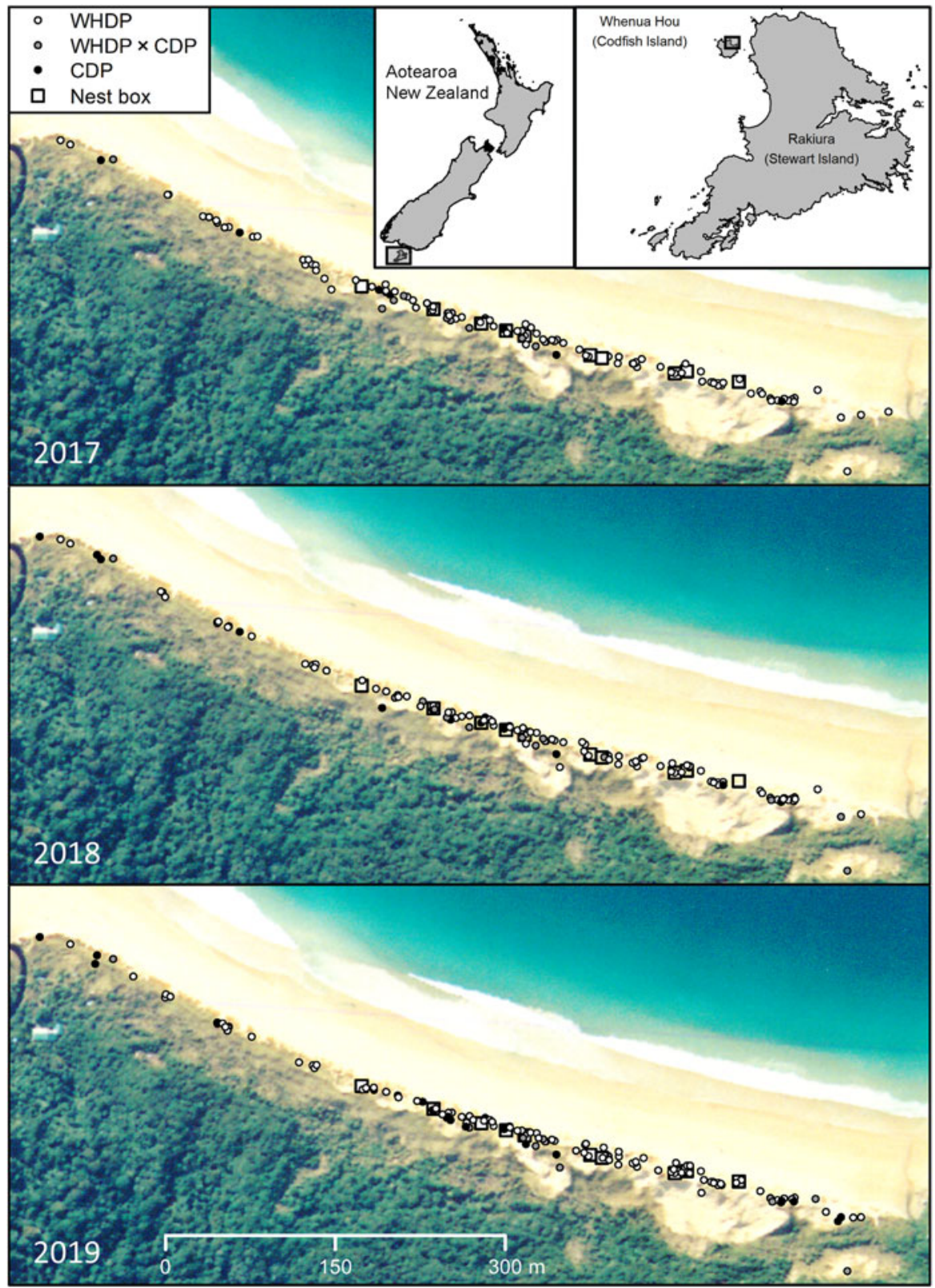

FIg. 1 Distribution of Whenua Hou diving petrel Pelecanoides whenuahouensis (WHDP), common diving petrel Pelecanoides urinatrix (CDP) and mixed burrows, and nest boxes in 2017,2018 and 2019.

Eggs were detected in 48 of the 62 monitored burrows in 2017 (apparent lay rate $=0.77$ ), in 57 of the 64 monitored burrows in 2018 (apparent lay rate $=0.89$ ), and in 62 of the 78 monitored burrows in 2019 (apparent lay rate $=0.79$ ), resulting in 167 Whenua Hou diving petrel burrows used in subsequent analyses. During each nest check, we recorded the phenological stage (egg, chick or fledged) and fate (dead or alive). These data were compiled in three capture history matrices showing whether each egg had hatched, chick had fledged, and was alive $(1=$ yes, $\mathrm{o}=$ no, NA $=$ not checked) each day. We assumed nests to be alive on first detection but otherwise treated the fate of eggs as unknown until a nest was either abandoned or the egg had hatched.

We estimated nest survival using a multi-stage nest survival model within a Bayesian framework (Schmidt et al., 2010; Converse et al., 2013). Our custom, multi-stage nest survival model allowed for (1) unknown transition and failure dates, (2) varying lengths of phenological stages among nests, (3) estimation of daily survival rates for two phenological stages (eggs and chicks), and (4) the estimation of fixed and random effects affecting the daily survival rate. Specifically, we fitted the data to a generalized linear mixed-effects model (GLMM) with a Bernoulli error term and a logit-link function:

$$
\begin{aligned}
\operatorname{logit}\left(D S R_{i, j}\right)= & \alpha_{D S R}+\beta_{\text {hatch }} \times \text { hatch }_{i, j}+\beta_{\text {sea }} \\
& \times \text { dist }_{i}+\beta_{W H D P} \times W H D P_{i}+\beta_{C D P} \\
& \times C D P_{i}+u_{\text {year }, y}
\end{aligned}
$$

where $D S R_{i, j}$ is the survival probability of nest $i$ on day $j$, $\alpha_{D S R}$ is the intercept, $\beta_{\text {hatch }}$ is the effect of transitioning from egg stage $\left(\right.$ hatch $\left._{i, j}=0\right)$ to chick stage $\left(\right.$ hatch $\left._{i, j}=1\right)$, 


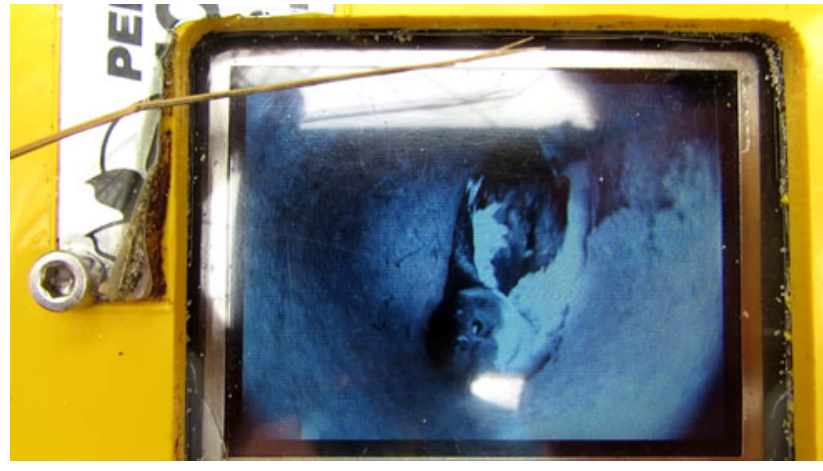

Plate 1 A burrowscope being inserted into a Whenua Hou diving petrel burrow.

$\beta_{\text {sea }}$ is the effect of distance to sea $\left(\right.$ dist $\left._{i}\right), \beta_{W H D P}$ is the effect of Whenua Hou diving petrel burrow density $\left(W H D P_{i}\right)$, $\beta_{C D P}$ is the effect of common diving petrel burrow density $\left(C D P_{i}\right)$, and $u_{\text {year, } y}$ is the annual random effect. We then estimated daily survival rate for the egg $\left(D S R_{\text {egg,y }}\right)$, and chick stage $\left(D S R_{\text {chick,y }}\right)$ per breeding season as:

$$
\begin{gathered}
\operatorname{logit}\left(D S R_{\text {egg,y }}\right)=\alpha_{D S R}+u_{\text {year }, y} \\
\operatorname{logit}\left(D S R_{\text {chick,y }}\right)=\alpha_{D S R}+\beta_{\text {hatch }}+u_{\text {year }, y}
\end{gathered}
$$

Subsequently we estimated nest survival during the egg $\left(S_{\text {egg,y }}\right)$ and chick $\left(S_{\text {chick,y }}\right)$ stage per breeding season as:

$$
\begin{gathered}
S_{\text {egg, } y}=D S R_{\text {egg, },}^{T_{\text {inc }}} \\
S_{\text {chick }, y}=D S R_{\text {chick }, y}^{T_{\text {rear }}}
\end{gathered}
$$

in which $T_{\text {inc }}$ and $T_{\text {rear }}$ are the estimated mean durations of the incubation and chick-rearing stages, respectively. Ultimately, we estimated overall nest survival per breeding season $\left(S_{y}\right)$ as:

$$
S_{y}=S_{\text {egg,y }} \times S_{\text {chick }, y}
$$

As we did not know exact dates of phenology events or duration of stages for all nests, missing values for hatching and fledging status were inferred by modelling the duration of each stage for each nest (Miller et al., 2017). We assumed these durations were normally distributed among nests with means $T_{i n c}$ and $T_{\text {rear }}$ and standard deviations $\sigma_{i n c}$ and $\sigma_{\text {rear }}$. We used mildly informative priors for these parameters: $\mathrm{N}[$ mean $=45$, precision $=0.1]$ for $T_{i n c}$ and $T_{\text {rear }}, \mathrm{U}[0,6]$ for $\sigma_{\text {inc }}$ and $\mathrm{U}[\mathrm{o}, 4]$ for $\sigma_{\text {rear }}$. We based our priors on the breeding phenology of the closely related South Georgian diving petrel Pelecanoides georgicus (Marchant \& Higgins 1990). We also used a mildly informative prior for $\alpha_{D S R}(\mathrm{~N}[5,1])$ but used vague priors for $\beta_{\text {hatch }}, \beta_{W H D P}, \beta_{C D P}$, and $\beta_{\text {sea }}(\mathrm{N}$ $[0,1])$ and for the standard deviation of the random effect $u_{\text {year }, y}(\mathrm{U}[0,1])$.

We measured dist $_{i}$ as the distance from the Whenua Hou diving petrel burrow to the highest springtide line per breeding season $(\mathrm{m})$. We measured $W H D P_{i}$ as the density of other Whenua Hou diving petrel burrows within a 3-m radius of the burrow (burrows per $\mathrm{m}^{2}$ ). Similarly, we measured $C D P_{i}$ as density of common diving petrel and mixed burrows within $3 \mathrm{~m}$ (burrows per $\mathrm{m}^{2}$ ). We $z$-transformed these three variables.

We fitted the model using the Bayesian updating software OpenBugs 3.2.3 (Spiegelhalter et al., 2014). The Markov Chain Monte Carlo (MCMC) algorithms make it possible to account for multiple sources of uncertainty, such as survival probability, timing, and duration of phenology stages, which are propagated into posterior distributions for parameters. We pooled two MCMC chains of 100,00o iterations, after a burn-in of 25,000 iterations that was sufficient to give convergence based on Gelman-Rubin statistics $(\hat{R}<1.05)$.

\section{Breeding phenology}

To quantify the timing and duration of Whenua Hou diving petrel breeding phenology, we monitored 25-30 burrows $(26-30 \%$ of the total population) daily between early September and late January each breeding season. We recorded arrival dates of birds based on when burrows were dug out by birds (burrows close in winter because of the movements of the dunes). We quantified the timing of subsequent breeding phenology events, including dates for laying, hatching, commencement of post-guard phase (i.e. as the first day a chick was left unattended by an adult), and fledging, using a burrowscope. We monitored burrows daily until we recorded a breeding phenology event, after which we ceased monitoring until a week before the next anticipated event. We initially predicted the timing of these events using published data on the closely related South Georgian diving petrel (Marchant \& Higgins, 1990). We used the timing of phenology events to delineate the phenological stages of courtship, incubation, chick-rearing (consisting of guard and post-guard stages) and the total breeding season, and to calculate the duration of these stages. We assessed the influence of interannual variation on timing and duration of breeding phenology stages using generalized linear models (GLMs) with a Gaussian error distribution and an identity-link function. In these GLMs we treated initiation date or duration per stage as the response variable and year as the explanatory variable. We first transformed initiation dates into a numerical variable (i.e. days since 1 September) and subsequently $z$-transformed initiation dates and durations.

\section{Burrow attendance}

As a proxy for Whenua Hou diving petrel feeding regimes, we quantified burrow attendance (i.e. visits per day) per phenological stage (i.e. courtship, incubation, guard and post-guard stages) using stick palisades. We assessed the influence of interannual variation per stage using GLMs 
with a quasi-binomial error distribution and a logit-link function. In these GLMs we treated visit per day per stage as the response variable and year as the explanatory variable. In addition, we assessed how burrow attendance during the post-guard stage changed over time using GLMs with a binomial error distribution and a logit-link function treating visits per day as the response variable and age (expressed as days-before-fledging) as the explanatory variable. We could not account for double feeds (i.e. both parents feeding the chick) using our stick palisade method. Thus, burrow attendance should be considered only a proxy for feeding regimes.

\section{Chick growth curves}

To generate Whenua Hou diving petrel chick growth curves (i.e. wing length and weight), we monitored 10 burrows between early December and late January each breeding season. To access chicks inside burrows, we installed 10 custom multi-storey nest boxes in existing burrows in early September 2017, before birds returned (Fig. 1, Supplementary Fig. 1; Fischer et al., 2018a). We selected burrows for nest box instalment if burrows belonged to successful breeders in 2015 and/or 2016, brood chambers had a depth of $<60 \mathrm{~cm}$, and burrows were $>10 \mathrm{~m}$ from the springtide line. We subjected chicks in nest boxes to daily measurements of weight $(\mathrm{g})$ and wing length (i.e. flattened wing chord; $\mathrm{mm}$ ) once they reached the post-guard stage until they fledged. Only four chicks fledged from a nest box (most pairs dug new brood chambers behind nest boxes).
We increased our sample size by taking measurements from all chicks accessible within natural burrows $(n=5)$. We also took measurements from all chicks caught just before fledging $(\mathrm{n}=80)$. We compared chick measurements with mean adult weight $(127 \mathrm{~g} ; \mathrm{n}=136)$ and mean adult wing length $(120 \mathrm{~mm} ; \mathrm{n}=111)$.

\section{Results}

\section{Nest survival}

The daily survival rate of Whenua Hou diving petrel eggs was estimated to be 0.992 ( $95 \%$ credible intervals $=0.988$ 0.995; Fig. 2a). The daily survival rate of chicks was estimated to be 0.998 (0.996-0.999). The duration of the incubation stage was estimated to be $\widehat{T_{\text {inc }}}=49.1$ (48.1-50.0) days and the duration of the chick-rearing stage was estimated to be $\widehat{T_{\text {rear }}}=46.9(46.5-47.3)$ days. Egg survival was estimated to be $0.686(0.551-0.796)$. Chick survival was estimated to be 0.890 (0.808-0.947; Fig. 2b). Nest survival from laying to fledging was estimated to be 0.611 (0.460-0.738; Fig. 2c). Mean distance to sea was $6.7 \mathrm{~m}$ (range $=0.1-20.7)$, mean Whenua Hou diving petrel density was 0.02 burrows per $\mathrm{m}^{2}$ (0.00-0.11) and mean common diving petrel density was 0.01 burrows per $\mathrm{m}(0.00-0.07)$. Estimates and $95 \%$ credible intervals for the effects of distance to sea $\left(\widehat{\beta_{\text {sea }}}=\right.$ $-0.01 ;-0.29,0.27)$, density of Whenua Hou diving petrel burrows $\left(\widehat{\beta_{W H D P}}=-0.01 ;-0.25,0.25\right)$, and density of
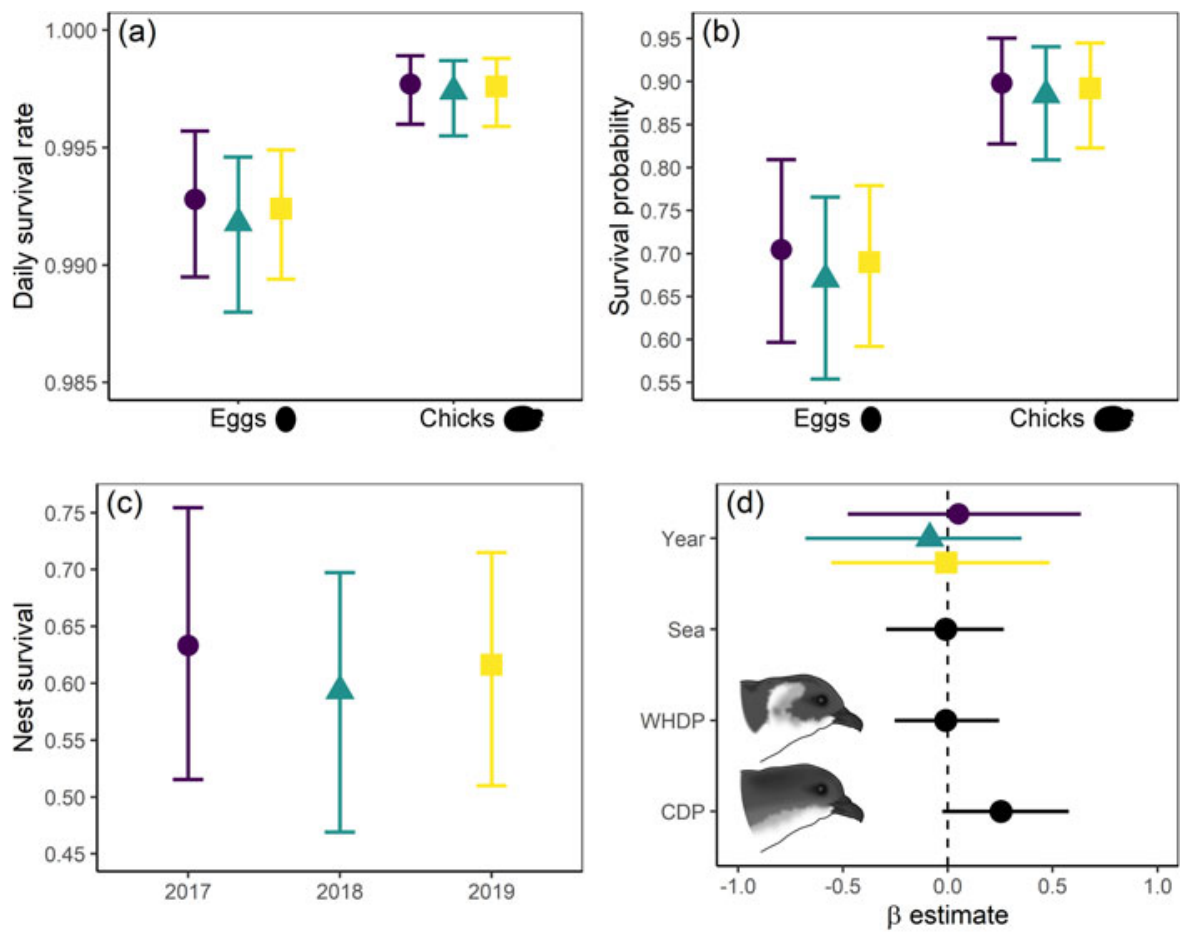

FIG. 2 Estimates and 95\% credible intervals for (a) daily survival rates of Whenua Hou diving petrel eggs and chicks, (b) probabilities of surviving the eggs and chick stages, (c) overall nest survival, and (d) slopes $(\beta)$ of $z$-transformed covariates affecting the logit of daily nest survival in the breeding seasons of 2017,2018 and 2019. Sea, effect of distance to sea; WHDP, effect of Whenua Hou diving petrel burrow density; CDP, effect of common diving petrel burrow 


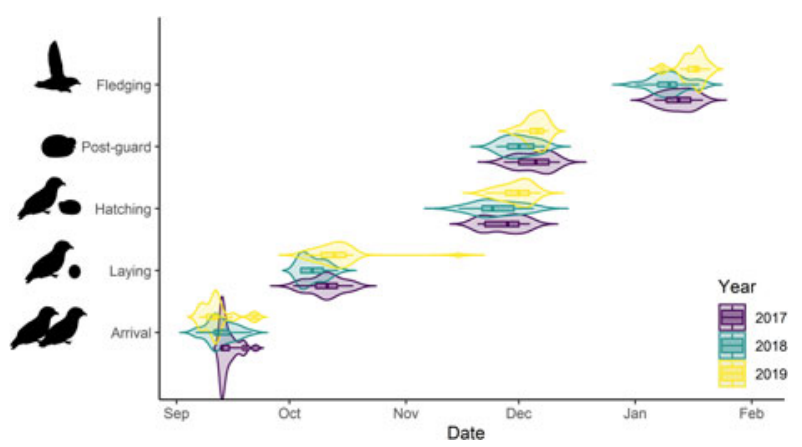

FIG. 3 Whenua Hou diving petrel phenology in the breeding seasons of 2017, 2018 and 2019.

common diving petrel burrows $\left(\widehat{\beta_{C D P}}=0.25 ;-0.03,0.58\right)$ did not indicate a clear impact on nest survival (Fig. 2d). There was also no apparent annual variation in survival. Twenty-four abandoned eggs were extracted from burrows to assess fertility, and 16 of these $(67 \%)$ were infertile.

\section{Breeding phenology}

On average, Whenua Hou diving petrels arrived at the colony on 13 September, eggs were laid on 10 October, chicks hatched on 27 November, post-guard stage commenced on 4 December, and fledging occurred on 13 January (Fig. 3). Phenology events were protracted and non-synchronous among burrows. Timing of breeding phenology varied slightly among breeding seasons. Specifically, arrival occurred slightly earlier in 2019 (estimates \pm SE: $\beta_{2018}=$ $\left.-0.50 \pm 0.26, \beta_{2019}=-0.80 \pm 0.25\right)$, laying and hatching occurred slightly later in $2019\left(\beta_{2018}=-0.53 \pm 0.28, \beta_{2019}=\right.$ $0.51 \pm 0.25$ and $\beta_{2018}=-0.31 \pm 0.30, \beta_{2019}=0.64 \pm 0.28$, respectively), post-guard commenced slightly earlier in $2018\left(\beta_{2018}=-0.88 \pm 0.33, \beta_{2019}=0.11 \pm 0.34\right)$, and fledging occurred slightly later in $2019\left(\beta_{2018}=-0.49 \pm 0.31, \beta_{2019}=\right.$ $0.81 \pm 0.27)$.
The average duration of breeding stages was as follows: courtship: 28 .o days; incubation: 48.2 days; chick guard stage: 8.2 days; post-guard stage: 39.1 days; resulting in 46.9 days for the total chick-rearing period. The total breeding season lasted 123.5 days. Duration of courtship stages, incubation stages, and total breeding periods varied slightly among breeding seasons. The courtship and incubation periods lasted slightly longer in 2019 (estimates \pm SE: $\beta_{2018}=-0.13 \pm$ $0.28, \beta_{2019}=0.86 \pm 0.25$ and $\beta_{2018}=-0.04 \pm 0.33, \beta_{2019}=0.76 \pm$ 0.29 , respectively), resulting in a longer breeding season $\left(\beta_{2018}=0.05 \pm 0.31, \beta_{2019}=1.13 \pm 0.28\right)$. Durations of the guard and post-guard stages were consistent among breeding seasons $\left(\beta_{2018}=0.41 \pm 0.36, \beta_{2019}=0.47 \pm 0.38\right.$ and $\beta_{2018}=-0.08 \pm 0.38$, $\beta_{2019}=0.22 \pm 0.39$, respectively).

\section{Burrow attendance}

Whenua Hou diving petrel burrow attendance was not uniform throughout the breeding season. Burrow attendance was lower during incubation (o.61 visits per day), compared to burrow attendance during courtship (o.83), guard (o.87), and post-guard (0.85) stages (Fig. 4a). Burrow attendance per stage varied slightly among seasons. Specifically, burrow attendance during courtship was higher in 2018 (estimates \pm SE: $\quad \beta_{2018}=1.04 \pm 0.29, \quad \beta_{2019}=-0.32 \pm 0.19$ ), burrow attendance during incubation was higher in 2019 $\left(\beta_{2018}=0.04 \pm 0.15, \beta_{2019}=0.37 \pm 0.14\right)$, burrow attendance during guard was lower in $2017\left(\beta_{2018}=1.07 \pm 0.39, \beta_{2019}=\right.$ $1.85 \pm 0.42)$, and burrow attendance during post-guard was higher in $2017\left(\beta_{2018}=-0.62 \pm 0.23, \beta_{2019}=-0.63 \pm 0.21\right)$. During the post-guard stage, visitation rates decreased over time from 0.94 visits per day in the early post-guard stage (40-20 days-before-fledging) to 0.65 visits per day during the last week before fledging (Fig. $4 \mathrm{~b}$ ). This decrease in visitation rates was more pronounced in $2019(\beta=-0.35 \pm$ $0.05)$ and $2018(\beta=-0.14 \pm 0.02)$ than in $2017(\beta=-0.03 \pm$ $0.01)$.
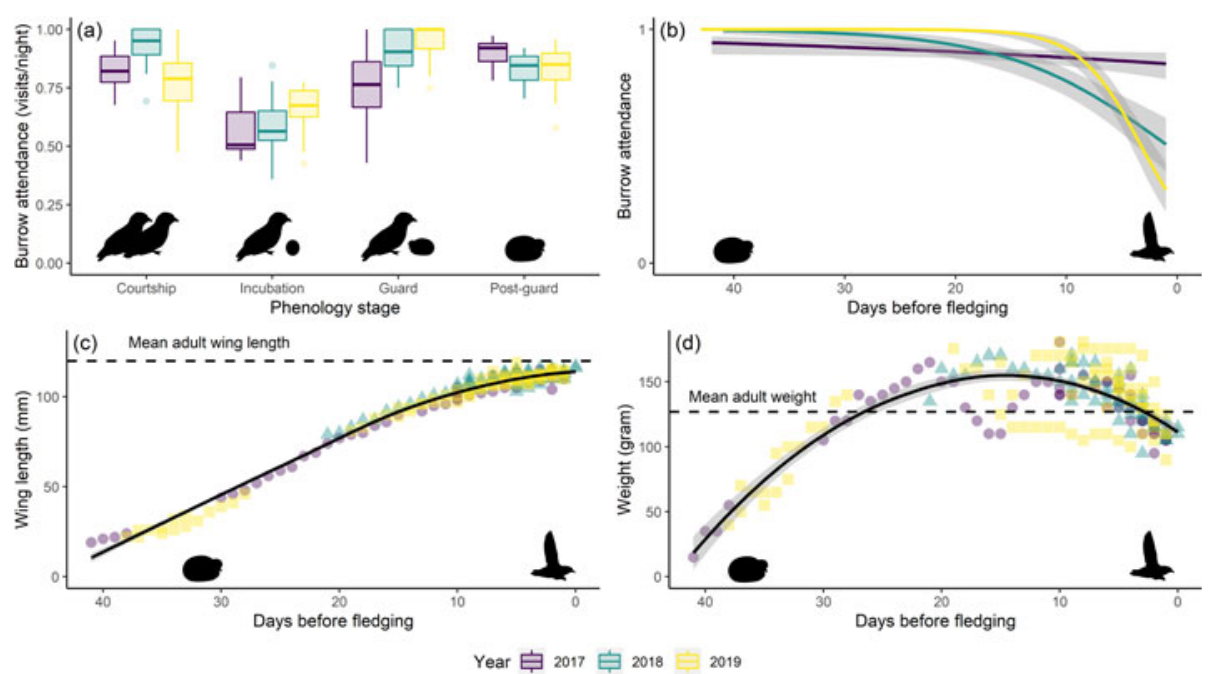

FIG. 4 Whenua Hou diving petrel burrow attendance across the breeding season (a), burrow attendance changes during the post-guard stage illustrated with generalized linear models (b), and chick growth curves of wing length (c) and weight (d) as illustrated by locally estimated scatterplot smoother curves. 


\section{Chick growth curves}

Wing lengths of Whenua Hou diving petrel chicks showed, on average, a gradual and consistent growth from $20 \mathrm{~mm}$ at 40 days before fledging until approaching a plateau of $111 \mathrm{~mm}$ (93\% of adult mean) around 7 days before fledging (Fig. 4 C). Maximum recorded wing length was $119 \mathrm{~mm}$ ( $99 \%$ of adult mean). Weight of chicks, on average, increased from $25 \mathrm{~g}$ at 40 days before fledging to a maximum of $148 \mathrm{~g}$ ( $117 \%$ of adult mean) between 20 and 10 days before fledging and subsequently decreased to $109 \mathrm{~g}$ ( $86 \%$ of adult mean) around fledging (Fig. 4d). Maximum recorded chick weight was $180 \mathrm{~g}(142 \%$ of adult mean).

\section{Discussion}

Our detailed study of nest survival and breeding biology has the potential to inform future translocations to establish a new breeding colony of the Critically Endangered Whenua Hou diving petrel. Firstly, by applying a novel Bayesian multi-stage nest survival model, we provide insights into nest survival. Estimates of demographic parameters such as nest survival are of vital importance when making structured decisions on conservation management (including translocations) in the face of uncertainty (Panfylova et al., 2019). For wide-ranging species such as petrels, nest survival, not juvenile or adult survival, will be the likely driver of translocation success, making nest survival estimates crucial for projecting future population trajectories at translocation sites. In addition, our estimates of the effects of parameters on nest survival are important for assessing the suitability of translocation sites. Although no parameters showed a clear impact on nest survival, these may have an influence at different exposure levels (e.g. nest survival may have been affected at higher common diving petrel burrow densities). As such, these parameters should still be considered when assessing translocation site suitability (Fischer et al., 2017).

Secondly, our findings on phenology, chick growth and nest survival help determine the ideal timeframe for collecting live chicks for translocations. As petrel chicks should be collected 1-6 weeks prior to fledging to prevent imprinting on the natal colony (Miskelly \& Taylor, 2004; Miskelly et al., 2009), collection of Whenua Hou diving petrel chicks should occur between early December and early January. As chicks reach their maximum weight 20-10 days before fledging and may thus be less susceptible to stress associated with translocations, the last week of December appears the ideal time to collect chicks of this species.

Thirdly, our growth curves will help select the best suited Whenua Hou diving petrel chicks for future translocations. Wing length combined with weight facilitates the estimation of age and condition of chicks (in days before fledging; Miskelly \& Taylor, 2004; Miskelly et al., 2009). Chicks selected for translocations should have a wing length of 75-105 mm and a weight of $>140 \mathrm{~g}$, as this combination will ensure the selection of healthy chicks at 20-10 days before fledging.

Fourthly, although the nest boxes were unpopular with adult Whenua Hou diving petrels, four chicks have fledged successfully from these boxes. Nest boxes did not appear to influence nest survival ( $4 / 8$ nest attempts inside nest boxes were successful). Since these nest boxes are designed specifically for this species (Fischer et al., 2018a), chicks can fledge successfully from them, and as access to chicks is crucial, the use of these nest boxes appears invaluable at future translocation sites.

Fifthly, our findings on burrow attendance and chick growth can inform post translocation feeding regimes for Whenua Hou diving petrel chicks. At the translocation site, chicks should be fed daily until 10-7 days before fledging to ensure chicks remain above mean adult weight. When translocated chicks approach fledging, feeds should be slowly reduced to every second day, provided chicks are at, or above, mean weight (Miskelly et al., 2009). We did not obtain species-specific information on meal size or diet. However, common diving petrel chicks have been successfully hand-reared using average meal sizes of $25 \mathrm{~g}$ (range 10-30; Miskelly et al., 2009). As common diving petrels have similar adult weights (110-150 g), these meal sizes may also be appropriate for Whenua Hou diving petrel chicks. Miskelly et al. (2009) and Miskelly \& Gummer (2013) have shown that petrel species will thrive on a diet of pureed sardines, regardless of their natural diet, and thus this diet may also be suitable for this species.

Whenua Hou diving petrel nest survival and breeding biology was largely unaffected by annual variation and therefore varying climatic conditions. The three breeding seasons in our study encompassed a range of climatic (El Niño Southern Oscillation; ENSO) conditions: Oceanic Niño Index was - 0.79 (La Niña) in 2017, 0.75 in 2018 (El Niño), and 0.43 (approaching ENSO neutral) in 2019 (NOAA, 2020). The slight differences in timing and duration of phenology appeared unrelated to ENSO conditions. The breeding season under neutral conditions (2019) was delayed and prolonged compared to other breeding seasons under more extreme and varying conditions. The apparent phenological insensitivity of Whenua Hou diving petrels to climatic conditions is not surprising as it mirrors insensitivity observed in many other seabirds (Keogan et al., 2018). Climatic variables, however, could have influenced burrow attendance (Quillfeldt et al., 2007). In 2017 (La Niña), burrow attendance was higher during the post-guard stage. Food supplies can change with climatic conditions (Schreiber \& Schreiber, 1984). Thus, adults in 2017 may have spent more time provisioning their chicks during the post-guard stage (Chastel et al., 1995).

The Whenua Hou diving petrel population is extremely small (194-208 adults; Fischer et al., 2020a) and room for error in management is slim. Further research is required 
prior to attempting translocations of this species. The appropriate cohort size and number of cohorts used for translocations must be estimated to minimize risks to the source population. The impact of translocations on the source colony could be quantified using population models. We provided a key parameter, nest survival, for such models, but juvenile and adult survival remain unknown and are currently under investigation (Fischer, 2020). Furthermore, infertility appeared a prevalent cause of egg failure. Although common diving petrels can have a similar rate of infertility (Richdale, 1945), the Whenua Hou diving petrel population probably suffered from a population bottleneck and represents a fraction of the historic genetic diversity (Wood \& Briden, 2008). As such, the selection of chicks for future translocations may benefit from including a measure of genetic diversity.

Translocations are a useful tool to combat the ongoing sixth mass extinction by restoring species and ecosystem functioning (Seddon et al., 2007, 2014). Translocations of petrels fit both conservation and restoration goals (Miskelly et al., 2009). We have provided most of the data required to inform future translocations. The Whenua Hou diving petrel is the only petrel species in Aotearoa New Zealand that breeds en masse in coastal dunes (Worthy, 1998) and is thus considered an ecosystem engineer (Fischer et al., 2019). The Predator Free 2050 programme (Russell et al., 2015) aims to eradicate seven species of invasive mammals from all of Aotearoa New Zealand by 2050. If this programme is successful, more habitat could become available for potential Whenua Hou diving petrel translocations. The information provided here may facilitate not only the conservation of a Critically Endangered species, but also the restoration of ecosystem function to a threatened habitat throughout Southern Aotearoa New Zealand.

Acknowledgements JHF was supported by a Victoria University of Wellington Doctoral Scholarship. Fieldwork was supported by the National Geographic Society (WW-249C-17), the Ornithological Society of New Zealand (Bird NZ Research Fund 2017, 2019), the Mohamed Bin Zayed Species Conservation Fund (Project 192520234), the Encounter Foundation, Forest and Bird (JS Watson Trust 2017), the Centre for Biodiversity and Restoration Ecology (Student Award 2017), and the Royal Society of New Zealand (Hutton Fund 2017). We thank Ngāi Tahu, the Whenua Hou Komiti, and Kaitiaki Rōpū for granting visits to Whenua Hou; the dozens of volunteers for their assistance in the field; Brooke Tucker for archaeological insights; Johannes Chambon, Jake Tessler and Dominique Filippi for technical support; Janne de Hoop, David Young and Johannes Chambon for providing artwork; and Ben Dilley, Colin Miskelly, Jason Preble and Grace Tocker for their comments.

Author contributions Study design, fieldwork: all authors; data analysis, writing: JHF, HUW, DPA.

\section{Conflicts of interest None.}

Ethical standards This research abided by the Oryx guidelines on ethical standards. All methods and protocols were approved by an institutional animal ethics committee (VUW AEC 23283 and VUW AEC 27621), the Department of Conservation (M1718/01, M1819/ 01, and M1920/02), the Whenua Hou Komiti, and Kaitiaki Rōpū.

\section{References}

Anderson, O.R.J., Small, C.J., Croxall, J.P., Dunn, E.K., Sullivan, B.J., Yates, O. \& Black, A. (2011) Global seabird bycatch in longline fisheries. Endangered Species Research, 14, 91-106.

Chastel, O., Weimerskirch, H. \& Jouventin, P. (1995) Body condition and seabird reproductive performance: a study of three petrel species. Ecology, 76, 2240-2246.

Cole, R. (2004) Summary of South Georgian Diving Petrel Field Observations for 2003/04, Codfish Island/Whenua Hou. Department of Conservation, Invercargill, New Zealand.

Converse, S.J., Royle, A., Adler, P.H., Urbanek, R.P. \& Barzen, J.A. (2013) A hierarchical nest survival model integrating incomplete temporally varying covariates. Ecology and Evolution, 3, 4439-4447.

Croxall, J.P., Butchart, S.E.M., Lascelles, B., Stattersfield, A.J., Sullivan, B., Symes, A. \& TAylor, P. (2012) Seabird conservation status, threats and priority actions: a global assessment. Bird Conservation International, 22, 1-34.

Deguchi, T., Suryan, R.M., Ozaki, K., Jacobs, J.F., Sato, F., Nakamura, N. \& BAlgh, G.R. (2014) Translocation and hand-rearing of the short-tailed albatross Phoebastria albatrus: early indicators of success for species conservation and island restoration. Oryx, 48, 195-203.

Dias, M.P., Martin, R., Pearmain, E.J., Burfield, I.E., Small, C., Phillips, R.A. et al. (2019) Threats to seabirds: a global assessment. Biological Conservation, 237, 525-537.

Einoder, L.D. (2009) A review of the use of seabirds as indicators in fisheries and ecosystem management. Fisheries Research, 95, 6-13.

Ellis, J.C. (2005) Marine birds on land: a review of plant biomass, species richness, and community composition in seabird colonies. Plant Ecology, 181, 227-241.

Fischer, J.H. (2020) Integrated conservation of the Whenua Hou diving petrel. $\mathrm{PhD}$ thesis. Victoria University of Wellington, Wellington, New Zealand.

Fischer, J.H., Chambon, J., Debski, I., Hiscock, J.A., Cole, R., Taylor, G.A. \& Wittmer, H.U. (2018a) Buffering artificial nest boxes for Procellariiformes breeding in exposed habitats: investigating effects on temperature and humidity. Notornis, 65, 35-41.

Fischer, J.H., Debski, I., Miskelly, C.M., Tennyson, A.J.D., Fromant, A., Tessler, J. et al. (2018b) Analyses of phenotypic differentiations between South Georgian Diving Petrel (Pelecanoides georgicus) populations reveal an undescribed and highlyendangered species from New Zealand. PLOS ONE, 13, eo197766.

Fischer, J.H., Debski, I., Taylor, G.A. \& Wittmer, H.U. (2018c) Nest site selection of South Georgia diving-petrels on Codfish Island (Whenua Hou), New Zealand: implications for conservation management. Bird Conservation International, 28, 216-227.

Fischer, J.H., Debski, I., Taylor, G.A. \& Wittmer, H.U. (2017) Assessing the suitability of non-invasive methods to monitor interspecific interactions and breeding biology of the South Georgian diving petrel (Pelecanoides georgicus). Notornis, 64, 13-20.

Fischer, J.H., McCauley, C.F., Armstrong, D.P., Debski, I. \& WitTMER, H.U. (2019) Contrasting responses of lizard occurrences to burrowing by a Critically Endangered seabird. Community Ecology, 20, 64-74.

Fischer, J.H., Taylor, G.A., Cole, R., Debski, I., Armstrong, D.P. \& Wittmer, H.U. (2020a) Population growth estimates of a threatened seabird indicate necessity for additional management 
following invasive predator eradications. Animal Conservation, 23, 94-103.

Fischer, J.H., Taylor, G.A, Debski, I. \& Wittmer, H.U. (202ob) Acoustic attraction system draws in competing seabird species. Notornis, 67, 568-572.

Grémillet, D., Ponchon, A., Paleczny, M., Palomares, M.L.D., Karpouzi, V. \& Pauly, D. (2018) Persisting worldwide seabird-fishery competition despite seabird community decline. Current Biology, 28, 4009-4013.

Holdaway, R.N., Jones, M.D. \& Athfield, N.R.B. (2003) Establishment and extinction of a population of South Georgian diving petrel (Pelecanoides georgicus) at Mason Bay, Steward Island, New Zealand, during the late Holocene. Journal of the Royal Society of New Zealand, 33, 601-622.

IUCN (2013) Guidelines for Reintroductions and other Conservation Translocations. Version 1.o. Species Survival Commission, Gland, Switzerland.

Jones, H.P. \& KRESS, S.W. (2012) A review of the world's active seabird restoration projects. Journal of Wildlife Management, 76, 2-9.

Jones, H.P., Tershy, B.R., Zavaleta, E.S., Croll, D.A., Keit t, B.S., Finkelstein, M.E. \& Howald, G.R. (2008) Severity of the effects of invasive rats on seabirds: a global review. Conservation Biology, 22, 16-26.

Keogan, K., Daunt, F., Wanless, S., Phillips, R.A., Walling, C., Agnew, P. et al. (2018) Global phenological insensitivity to shifting ocean temperatures among seabirds. Nature Climate Change, 8, 313-318.

Marchant, S. \& Higgins, P.J. (1990) Handbook of Australian, New Zealand and Antarctic Birds. Volume 1, Ratites to Ducks, Part A, Ratites to Petrels. Oxford University Press, Melbourne, Australia.

McClelland, P.J. (2002) Eradication of Pacific rats (Rattus exulans) from Whenua Hou Nature Reserve (Codfish Island), Putauhinu and Rarotoka Islands, New Zealand. Turning the Tide: The Eradication of Invasive Species, 27, 173-181.

Miller, M.W., Leech, D.I., Pearce-Higgins, J.W. \& Robinson, R.A. (2017) Multi-state, multi-stage modelling of nest success suggests interaction between weather and land-use. Ecology, 98, 175-186.

Miskelly, C.M. \& Gummer, H. (2013) Attempts to anchor pelagic fairy prions (Pachyptila turtur) to their release site on Mana Island. Notornis, 60, 29-40.

Miskelly, C.M. \& TAYLOR, G.A. (2004) Establishment of a colony of common diving petrels (Pelecanoides urinatrix) by chick transfers and acoustic attraction. Emu, 104, 205-211.

Miskelly, C.M., Taylor, G.A., Gummer, H. \& Williams, R. (2009) Translocations of eight species of burrow-nesting seabirds (genera Pterodroma, Pelecanoides, Pachyptila and Puffinus: family Procellariidae). Biological Conservation, 142, 1965-198o.

NOAA (2020) National Oceanic and Atmospheric Administration Climate Prediction Database Repository. origin.cpc.ncep.noaa.gov/ products/analysis_monitoring/ensostuff/ONI_v5.php [accessed 1 April 2020].

Orben, R.A., O’Connor, A.J., Suryan, R.M., Ozaki, K., Sato, F. \& DEGUCHI, T. (2018) Ontogenic changes in at-sea distributions of immature short-tailed albatrosses Phoebastria albatrus. Endangered Species Research, 35, 23-27.

Orwin, K.H., Wardle, D.A., Towns, D.R., John, M.G., Bellingham, P.J. \& Jones, C. (2016) Burrowing seabird effects on invertebrate communities in soil and litter are dominated by ecosystem engineering rather than nutrient addition. Oecologia, 180, 217-230.

Osborne, P.E. \& SEddon, P.J. (2012) Selecting suitable habitats for reintroductions: variation, change and the role of species distribution modelling. In Reintroduction Biology: Integrating Science and
Management (eds J.G. Even, D.P. Armstrong, K.A. Parker \& P.J. Seddon), pp. 73-104. Blackwell Publishing, Oxford, UK.

Otero, X.L., de la Peña-Lastra, S., Pérez-Alberti, A., Ferreira, T.O. \& Huerta-Diaz, M.A. (2018) Seabird colonies as important drivers in the nitrogen and phosphorus cycles. Nature Communications, 9, 246.

Panfylova, J., Ewen, J.G. \& Armstrong D.P. (2019) Making structured decisions for reintroduced populations in the face of uncertainty. Conservation Science and Practice, 1, e9o.

Priddel, D., Carlile, N. \& Wheeler, R. (2006) Establishment of a new breeding colony of Gould's Petrel (Pterodroma leucoptera leucoptera) through the creation of artificial nesting habitat and the translocation of nestlings. Biological Conservation, 128, 553-563.

Quillfeldt, P., Strange, I.J. \& Masello, J.F. (2007) Sea surface temperatures and behavioural buffering capacity in thin-billed prions Pachyptila belcheri: breeding success, provisioning and chick begging. Journal of Avian Biology, 38, 298-308.

Richdale, L.E. (1945) Supplementary notes on the diving petrel. Transactions of the Royal Society of New Zealand, 75, 42-53.

Rodríguez, A., Arcos, J.M., Bretagnolle, V., Dias, M.P., Holmes, N.D., Louzao, M. et al. (2019) Future directions in conservation research on petrels and shearwaters. Frontiers in Marine Science, 6, 94.

Rodriguez, A., Holmes, N.D., Ryan, P.G., Wilson, K.J., Faulquier, L., Murillo, Y. et al. (2017) Seabird mortality induced by land-based artificial lights. Conservation Biology, 31, 986-1001.

Russell, J.C., Innes, J.G., Brown, P.H. \& Byrom, A.E. (2015) Predator-free New Zealand: conservation country. BioScience, $65,520-525$.

Schmidt, J.H., Walker, J.A., Lindberg, M.S., Johnson, D.S. \& Stephens, S.E. (2010) A general Bayesian hierarchical model for estimating survival of nests and young. Auk, 127, 379-386.

Schreiber, R.W. \& Schreiber, E.A. (1984) Central Pacific seabirds and the El Niño Southern Oscillation: 1982 to 1983 perspectives. Science, 225, 713-716.

Seddon, P.J., Armstrong, D.P. \& Malony, R.F. (2007) Developing the science of reintroduction biology. Conservation Biology, 21, 303-312.

Seddon, P.J., Griffiths, C.J., Soorae, P.S. \& Armstrong, D.P. (2014) Reversing defaunation: restoring species in a changing world. Science, 345, 406-412.

Shaffer, S.A., Tremblay, Y., Weimerskirch, H., Scott, D., Thompson, D.R., SAGar, P.M. et al. (2006) Migratory shearwaters integrate oceanic resources across the Pacific Ocean in an endless summer. Proceedings of the National Academy of Sciences USA, 103, 12799-12802.

Spiegelhalter, D., Thomas, A., Best, N. \& Lunn, D. (2014) OpenBUGS User Manual, Version 323. Bugs, Cambridge, UK.

Vousdoukas, M.I., Ranasinghe, R., Mentaschi, L., Plomaritis, T.A., Athanasiou, P., Luijendijk, A. \& Feyen, L. (2020) Sandy coastlines under threat of erosion. Nature Climate Change, $10,260-263$.

Wood, J.R. \& Briden, S. (2008) South Georgian diving petrel (Pelecanoides georgicus) bones from a Maori midden in Otago Peninsula, New Zealand. Notornis, 55, 46-47.

Worthy, T.H. (1998) Fossils indicate Pelecanoides georgicus had large colonies at Mason Bay, Stewart Island, New Zealand. Notornis, 45, 229-246.

Žydelis, R., Small, C. \& French, G. (2013) The incidental catch of seabirds in gillnet fisheries: a global review. Biological Conservation, $162,76-88$ 